\title{
USO DE CLOBAZAN EN EPILEPSIAS DE DIFICIL CONTROL EN NINOS
}

\author{
PATRICIA CAMPOS O.*
}

\begin{abstract}
RESUMEN - Se presenta el estudio de 20 pacientes en edad pediátrica con diagnóstico de epilepsia de difícil control, en los cuales fue usado el clobazan como terapia anticonvulsivante coadyuvante, vistos entre Enero-1986 y Julio-1990 en el consultorio externo de Neuropedia. tría del Hospital General Base Cayetano Heredia y práctica privada, con un seguimiento entre 6 y 45 meses. La etad de inicio para la epilepsia fue en promedio 22 meses y la forma mas frecuente los sindromes de epilepsia generalizada, tanto secundaria como idiopática. Se obtuvo disminución de frecuencia de crisis en rango significativo en $60 \%$ de los pacientes con control completo en $35 \%$ del total, habiendose encontrado los mejores resultados en epilepsias generalizadas secundarias tipo Lennox-Gastaut y West. Se encontró fenómeno de tolerancia en $25 \%$ y efecto psicotrópico en tres pacientes. Sugerimos el uso de esta 1,5 benzodiazepina en epilepsias de dificil control en niños, en nuestro medio.
\end{abstract}

PALAVRas-CHAVE: epilepsia, infância, difícil controle, clobazan.

Use of clobazam in hard control epilepsy in children

SUMMARY - We present the study of 20 pediatric patients with difficult controlled epilepsy in which we used clobazam as adjuvant anticonvulsant therapy between January 1986 and July 1990 at the outpatients neuropediatric facility of the Hospital Genertal Base Cayetano Heredia and the private practice, with 6 to 45 months follow up. The mean age for the onset of the epilepsy was 22 months and the most frequent kind was the generalized epilepsy syndrome both secondary and idiopatic. It was obtained significant decrease of seizures in $60 \%$ of the patients with a complete control in $35 \%$ of the total. It was found the best outcome in secondary generalized epilepsies Lennox-Gastaut and West types. It was found tolerance phenomenọ in $25 \%$, and psychotropic effect in three patients. We suggest the use of this 1,5 benzodiazepine drug in difficult controlled epilepsies in children of our region.

KEY WORDS: epilepsy, infancy, hard control, clobazam.

Aproximadamente $20 \cdot 30 \%$ de las epilepsias en cualquier edad de la vida, especialmente en niños, son refractarias a tratamiento, constituyendo las llamadas epilepsias de difícil control y generalmente haciendo parte de sindromes convulsivos mixtos bien caracterizados. Desde 1965, con la introducción de las 1,4 benzodiazepinas en el arsenal terapeútico, se ha venido obteniendo cada vez mejor control de crisis, sobre todo cuando usadas en status epilepticus y en crisis menores (haciendo o no parte de sindromes epilépticos especificos) $2,9,14,15,19$, con el inconveniente de sedación como efecto colateral. Mas de 10 años después, en Francia, fue introducida como anticonvulsivante una 1,5 bezodiazepina (clobazan)12, la que viene teniendo un rol cada vez mas importante como fármaco coadyuvante, con controles totales de crisis en rangos de $12-55 \%$ y con mucho menor efecto de sedación.

* Profesora Asociada, Departamento de Neurología, Universidad Peruana Cayetano Heredia, Lima. Aceite: 05-março-1992.

Dra. Patricia Campos O. - Neuropediatria, Hospital General Base Cayetano Horedia - Apartado 5045 - Lima - Perú. 
Fl objetivo de presente estudio es analisar la experiencia a lo largo de 45 meses en el uso de clobazan como terapia coadyuvante en epilepsias de difícil control en niños. Adicionalmente, es uno de los pocos estudios realizados al respecto en Latinoamérica y con un tiempo de seguimiento mavor de 3 años.

\section{CASUISTICA Y METODOS}

El estudio se realizó entre Enero-1986 y Julio-1990 en 21 pacientes ambulatorios, tanto del consultorio externo de Neuropediatria del Hospital General Base Cayetano Heredia como de la práctica privada, que fueron vistos consecutivamente hasta Diciembre-1989 con el diagnóstico de epilepsia de difícil control, definida como epilepsia refractaria a tratamiento con tiempo de instalación mínimo de 4 meses, sin control apesar de niveles séricos de anticonvulsivantes adecuados y seriados durante los últimos 6 meses y usando 1 ó 3 drogras antiepilépticas consideradas de primer orden para los diferentes tipos de crisis presentadas. Se excluyó un paciente, el mismo que sólo volvió a un control dentro de los 6 primeros meses.

La frecuencia inicial de crisis fue catalogada en 3 grupos aleatoriamente: grupo A, mas de 7 crisis por día; grupo B, mas de 7 crisis por semana, grupo C, mas de 7-10 crisis por mes. El tiempo de seguimiento total varió entre 6 meses y 4 años 6 meses y el tiempo de uso de clobazan entre 6 y 45 meses (3 años 9 meses). Se usó el clobazan V.O. en dosis de $1-1.5 \mathrm{mg} / \mathrm{kg} / \mathrm{dia}$, generalmente en una sola toma diaria por la noche. Cinco de los pacientes lo recibieron en 3 dosig diarias.

Todos los pacientes fueron controlados con dosajes séricos de los otros anticonvulsivantes en forma seriada y sometidos por lo menos a un EEG. Se procedió además a la realización de diferentes exámenes auxiliares para determinar la etiología de la epilepsia.

\section{RESULTADOS}

EH número total de pacientes se dividió por igual entre hombres (50\%) y mujeres (50\%). La edad de la primera consulta varió entre 4 meses y 12 años, habiendo 7 (35\%) menores de 1 año. La edad de inicio de las crisis fue entre 2 meses y 7 años 6 meses (22 meses en promedio), habiendo empezado la epilepsia en 11 (55\% antes del primer año de vida.

En relación a formas de epilepsia, 18 (90\%) correspondieron a epilepsias generalizadas, de las cuales 11 (61\%) llenaban los criterios para sindrome de Lennox-Gastaut y 7 (39\%) eran espasmos infantiles; de los restantes, uno correspondió a una epilepsia parcial (forma límbica) y otro fue una epilepsia mixta con retardo mental (Tabla 1).

Tabla 1. Distribución de pacientes por formas de epilepsia y etiología.

\begin{tabular}{|c|c|c|c|c|}
\hline \multirow{2}{*}{\multicolumn{2}{|c|}{ Formas }} & \multicolumn{3}{|c|}{ Ettiología } \\
\hline & & Primaria & Secundaria & Total \\
\hline \multicolumn{5}{|c|}{ I. Generalizada } \\
\hline & Lennox-Gastaut & 2 & 9 & 11 \\
\hline & West & - & 7 & 7 \\
\hline \multicolumn{5}{|c|}{ II. Parciales } \\
\hline & Simples límbica & - & 1 & 1 \\
\hline III. & Mixta & 1 & 一 & $\mathbf{1}$ \\
\hline \multicolumn{2}{|c|}{ Total } & $3(15 \%)$ & $17(85 \%)$ & $20(100 \%)$ \\
\hline
\end{tabular}

La etiología de las crisis se dividió en: idiopática en 3 casos (15\%) y secundaria en 17 (85\%). (Tabla 2). De las secundarias el mayor porcentaje correspondió a secuelas de encefalopatía hipóxico-isquémica en el periodo neonatal en 8 (47\%), seguida de malformaciones congénitas del sistema nervioso central (SNC) en 4 (23.5\%), secuela de infecciones del SNC en 4 (23.5\%) y probłable secuela de infección intrauterina crónica en 1 (6\%). 
En relación a frecuencia de crisis tuvimos 10 pacientes en el grupo A (50\%), 6 en el B (30\%) y 4 en el C (20\%).

Eil exámen neurológico fue sin localización únicamente en 5 pacientes (25\%); los 15 restantes, todos tuvieron una $u$ otra forma de localización neurológica. Practicamente todos presentaron retardo mental, el cual fue severo en $10(52.6 \%)$, moderado en $6(31.5 \%)$ leve en 3 (15.9\%); hubo una sola paciente con CI fronterizo.

El EEG inicial, realizado en todos ellos dentro de la primera o segunda semana de acudir a la consulta, fue anormal en todos, con actividad paroxismal en 19 (95\%) y persiscente actividad lenta de base en 1 paciente, en el cual a pesar de repetidos trazados no se observó actividad paroxismal. El tipo de actividad paroxismal fue generalizada en 16 (90\%) y focal en $3(10 \%)$. La actividad generalizada correspondió a hipsarritmia con y sin salvas de supresión en 9 pacientes, punta-onda menor de $3 \mathrm{~Hz}$ en $6 \mathrm{y}$ multifocal en 1 . La actividad focal estuvo representada por un paciente de cada una de las siguientes localizaciones: rolándica, temporal anterior y hemisférica (Cuadro 2).

Tabla 2. Distribución de anormalidades EEG.

\begin{tabular}{|c|c|c|c|}
\hline & & No & $\%$ \\
\hline Actividad paroxismal & & 19 & 95 \\
\hline \multicolumn{4}{|l|}{ generalizada } \\
\hline hipsarritmia & 9 & & \\
\hline punta-onda $<3 \mathrm{~Hz}$ & 6 & & \\
\hline multifocal & 1 & & \\
\hline \multicolumn{4}{|l|}{ focal } \\
\hline rolândica & 1 & & \\
\hline temporal anterior & 1 & & \\
\hline hemisférica & 1 & & \\
\hline \multicolumn{4}{|l|}{ Lentificación actividad } \\
\hline de base & & 1 & $\mathbf{5}$ \\
\hline
\end{tabular}

Dos pacientes (10\%) recibian una droga (valproato de sódio ó carbamazepina), 12 (60\%) dos drogas y 6 (30\%) tres drogas anti-convulsivantes; la mayoría de las combinaciones terapeuticas usaban valproato de sodio. Todos ellos tenían niveles séricos de anticonvulsivantes en rangos terapeuticos al momento de iniciar terapia coadyuvante con clobazan.

Luego de iniciado el tratamiento, el porcentaje de mejoría (disminución de frecuencia de crisis) a las 4 semanas fue de $25 \%$ en 2 pacientes, $50 \%$ en $6,75 \%$ en 5 y $100 \%$ en 7 ; lo que hace un control mayor del 50\% en 12 pacientes (60\%). Desde el punto de vista de tipos de epilepsia el mejor control se obtuvo en las formas generalizadas secundarias (9/20 pacientes), especialmente sindromes de Lennox-Gastaut y West.

En el seguimiento a los 4 meses, 11 pacientes (55\%) permanecieron con igual porcentaje de control (en este grupo estando incluidos los 7 con control total), 3 continuaron mejorando alcanzando uno de ellos control total, y 1 empeoró nitidamente la frecuencia de crisis. Se presentó tolerancia en 5 pacientes (25\%).

Dentro de las 4 primeras semanas 2 pacientes presentaron irritabilidad y 1 somnolencia leve, que no fueron motivo para suspender el tratamiento. Después de los 4 meses se suspendió el tratamiento en 5: 2 por iniciativa de la madre, 1 por aumento de la frecuencia de la crisis, 1 por somnolencia excesiva que no la había presentado antes y que no pudo ser atribuida a los otros anticonvulsivantes y 1 por problema respiratorio (aumento de secreciones bronquiales). 
De los 15 racientes que continuaron recibiendo tratamiento al año, 1 volvió a incrementar nuevamente la frecuencia de crisis siendo reemplazado el clobazan por otra benzodiazepina y 14 permanecieron con el mismo grado de control. En el seguimiento posterior hubo otro paciente con iguales características en el que también se cambió a otra benzodiazepina. Hasta los 3 años de seguimiento la tolerancia aumentó para $35 \%$.

\section{COMEN'TARIOS}

Desde la introducción del clobazan como anticonvulsivante por Gastaut y col.12,13 hasta la actualidad, éste ha seguido probando su eficacia como terapia coadyuvante, especialmente en epilepsias de dificil control $5,1,11,1^{\prime} /, 18$, epilepsia catamenial $10 \mathrm{y}$, en los últimos ańos, en status epilépticus 22 .

Revisando el porcentaje de control obtenido en relación al tipo de crisis vemos que en 1971 Péchandre y col.18 obtuvieram $80 \%$ de disminución de frecuencia de crisis, especialmente en pacientes con sindrome de Lennox-Gastaut. Shimizu y col.21 obtuvieron control total o parcial en $50 \%$ de pacientes con crisis generalizadas. Em 1983, Allen y col.1 se referian básicamente al control de crisis parciales $y$, un año mas tarde en Latinoamérica, Figueroa y col.11 obtenian disminución de frecuencia de crisis en $72 \%$ de pacientes sobre todo en los que tenian crisis parciales. En años recientes Farrell y col.9 reportaram con. trol parcial en 11/17 pacientes $y$, en el seguimiento mayor de 3 meses, llegaron a controlarse completamente $20 \%$, principaimente los que tenían crisis menores. Munn y col.17 encontraram alrededor de $40 \%$ de control parcial en todo tipo de crisis. Cornaggia y col.4 obtuvieram $65 \%$ de mejoría tanto en crisis parciales como generalizadas. Shimizu y col. ${ }^{1}$, Gastaut y col.13, Callaghan y $\operatorname{col}^{3}$ y Diez Cuervos y col.6 son los que encuentran grupos de pacientes con control total de crisis, para éstes últimos hubo $\mathbf{5 0} \%$ de pacientes con sindrome de LennoxGastaut con control total.

En nuestra muestra encontramos mejoria de frecuencia de crisis entre 25-75\% para 13/20 pacientes, lo que constituye un control de $60 \%$ en mas de mitad de ellos, datos en relación con lo referido en la literatura; ya para el $35 \%$ de los pacientes los datos se asemejan a los de autores mencionados, con un control total de las crisis que, además, permaneció invariable en el seguimiento a largo plazo.

El llamado «efecto de exhaustión» por Gastaut 12, hoy llamado fenómeno de tolerancia, es una característica común a muchas benzodiazepinas $\mathrm{y}$ fuera encontrado por dicho autor en alrededor de un tercio de pacientes y presentándose pocas semanas después de iniciado el tratamiento. En la literatura revisada, fue encontrado fenómeno de tolerancia entre $12-77 \%$ y en un tiempo promedio de 3 meses $y$ medio (variando entre 1 y 18 meses) $1,4,6,11,17,21$. La tolerancia encontrada para nuestros pacientes fue de $25 \%$, observada alrededor del cuarto mes de iniciado el tratamiento, y aumentó para $35 \%$ en el seguimiento. El rango de variación descrito en la literatura posiblemente pueda explicar el porque un porcentaje apreciable de nuestros pacientes permanecieron con un control total o invariable en el seguimiento a largo plazo.

En relación a doisis usadas y horario de administración ésta varió entre 0.3-2 $\mathrm{mg} / \mathrm{Kg} /$ día para los diferentes autores, siendo administrada en 1, 263 tomas al día. Munn y col.17 reportam dosis de $0.33-0.71 \mathrm{mg} / \mathrm{Kg} /$ día y son los únicos que atribuyen un mayor porcentaje de mejoría a mayores dosis. En nuestra casuística, el rango usado ya fue mencionado y solamente un paciente usó $2 \mathrm{mg} / \mathrm{Kg} /$ día.

La sedación o sonnolencia es porcentualmente el efecto indeseable mas frecuentemente encontrado pero que, no necesariamente, obligó a retirar el tratamiento. Otros efectos secundarios descritos han sido irritabilidad, babeo constante, ataxia, aumento de secreciones bronquiales y transtornos del ciclo vigilia sueño 16. Estos efectos inclusive mejoraron al disminuir las dosis o únicamente con el correr de los días 11. En nuestra casuística la somnolencia, aunque presente, no impidió la continuación del tratamiento y los otros efectos colaterales descritos se presentaron en un rango mucho menor.

El efecto psicotrópico, descrito por Scott y Moffett 20 y por Trimble y Thompson 23 ha sido referido en la serie de Farrell $\mathrm{y}$ col. 9 como aumento del 
alerta en 34 pacientes, disminución de la hiperactividad en 19, además de mejoria de la marcha y postlira en 20. Nosotros no cuantificamos con test la mejoría en la atención pero clínicamente fue notoria la mejoría del contacto con el ambiente y del rendimiento escolar, además de disminución de hiperactividad en 3 pacientes: una niña con sindrome de Lennox-Gastaut secundario y retardo mental severo que actualmente acude y con buena performance a un centro de educación especial, un niño con epilepsia parcial forma límbica y un adolescente con epilepsia mixta idiopática y retardo mental leve.

Conclusiones - (1) Nuestra casuística aunque pequeña constituye una de las pocas en Latinoamérica con seguimiento a largo plazo mayor de 3 años. (2) Con el uso de clobazan como terapia antiepiléptica coadyuvante hemos conseguido una disminución de frecuencia de crisis en rango significativo en el $60 \%$ de nuestros pacientes, habiento tenido $35 \%$ de ellos totalmente controlados. (3) En relación al tipo de crisis los mejores resultados los hemos obtenido en epilepsias generalizadas secundarias, especialmente sindromes de Lennox-Gastaut y West. (4) El efecto de tolerancia fue encontrado para el 25\% de nuestros pacientes alrededor del cuarto mes de tratamiento, subiendo para $35 \%$ en el seguimiento mayor de 1 año. (5) Los efectos colaterales de la medicación de modo general no obligaron a suspensión de tratamiento. (6) Fue posible verificar clínicamente efecto psicotrópico en tres pacientes. (7) Creemos que, a la luz de los resultados obtenidos, podemos sugerir la utilización del clobazan como terapia coadyuvante en epilepsias de difícil control en niños en nuestro medio.

\section{REFERTECIAS}

1. Allen JW, Oxley J, Robertson MM, Trimble MR, Richens A, Jarad SM. Clobazam as adjuntive treatment in refractory epilepsy. Br Med $\mathrm{J}$ 1983, 286:1246-1247.

2. Bensch J, Blennow G, Ferngren H, Gamstorp I, Herrlin K-M, Kubista J, Arvidsson A, Dahlström H. A double-blind study of clonazepam in the treatment of therapy resistent epilepsy in children. Dev Med Child Neurol 1977, 19:335-342.

3. Callaghan N, Goggin I. Clobazam as adjuntive treatment in drug resistant epilepsy: report on an open prospective study. Ir Med J 1984, 77:240-244.

4. Coruaggia CM, Cattabeni G, Cerisola N, Leozzapa C, Mascetti PL, Massironi R, Porro G. Manghi E. Intractable epilepsies: an open trial with clobazam. Acta Neurol 1989, 11:1-8.

5. Critchley E, Vakil SD, Hayward H, Owen M, Cocks A, Freewantle NP. Double-blind clinical trial of clobazam in refractory epilepsy. $J$ S Soc Med 1981, 43:159-163.

6. Diez Cuervo A, Sanchez Caro J, Manrique M, Rodriguez Ramos P, Coullant JA, Perez Serrano J. Clobazan en politerapia de epilepsia de dificil control. Phronesis 1982, 3:53-58.

7. Dulac D, Figueroa D, Rey E, Arthuis M. Monotherapie par le clobazam dans les epilepsies de l'enfant. Presse Med 1983, 12:1067-1069.

8. Farrell $K$. Benzodiazepines in the treatment of children with epilepsy. Epilepsia 1986, 27 (Suppl 1): S45-S51.

9. Farrell K, Jan JE, Julian JV, Betts TA, Wong PK. Clobazam in children with intractables seizures. Epilepsia 1984, 25:657.

10. Feely M, Calvert R, Gibson J. Clobazam in catamenial epilepsy. Lancet 1982, 2:71-73,

11. Figueroa D, Adlerstein L, Manterola A. Clobazan en epilepsias refractarias del niñu. Rev Chil Pediat 1984, 55:401-405.

12. Gastaut H. Proprietes antiepileptiques exceptionnelles et meconnues d'un anxiolytique du commerce, le clobazam. Concours Med 1978, 100:3697-3701.

13. Gastaut H, Low MD. Antiepileptic properties of clobazam, a 1,5-benzodiazepine, in man. Epilepsia 1979, 20:437-446.

14. Gibbs F, Anderson E. Treatment of hipsarhythmia and infantile spams with a Librium analogue. Neurology 1965, 15:1173-1176.

15. Hanson RA, Menkes JH. A new anticonvulsant in the management of minor motor seizures. Dev Med Child Neurol 1972, 14:3-14.

16. Koeppen D, Baruzzi A, Capozza M, Chauvel P, Courjon J, Favel P, Lorenz H, Oller Ferrer-Vidal L, Procaccianti G, Fucquoy-Ponsar M, Sallou C, Sideri G, Trettier S, Weber M, Wolf $P$. Clobazam in therapy-resistant patients with partial epilepsy: a doubleblind placebo-controlled crossover study. Epilepsia 1987, 28:495-506.

17. Munn $R$, Camfield P, Camfield C, Dooley J. Clobazam for refractory childhood seizure disorders: a valuable supplementary drug. Can J Neurol Sci 1988, 15:406-408. 
18. Pechandre JC, Beudin P, Devize JL, Gilbert J. Use of clobazam as antiepileptic in the Lennox-Gastaut syndrome. Encephale 1981, $7: 181-190$.

19. Robertson M. Current status of the 1,4 and 1,5 benzodiazepines in the treatment of epilepsy: the place of clobazam. Filepsia 1986, 27 (Suppl 1): S7-S41.

20. Scott DF, Moffett A. On the anticonvulsant and psychotropic properties of clobazam: a preliminary study. Epilepsia 1986, 27 (Suppl 1): S42-S44.

21. Shimizu H, Abej, Futagi $Y$, Onoe S, Tagawa T, Mimaki T, Yamatodani A, Kato M, Kamio $M$, Sumi $\mathbf{K}$, Sugita $\mathbf{T}$, Yabuuchi $H$. Antiepileptic effects of clobazam in children. Brain Dev 1982, 4:57-62.

22. Tinuper P, Aguglia $O$, Gastaut $H$. Use of clobazam in certain forms of status epilepticus and in startle-induced epileptic seizures. Epilepsia 1986, 27 (Suppl 1): S18-S26.

23. Trimble MR, Thompson PJ. Anticonvulsant drugs: cognitive functions and behavior. Epilepsia 1983, 24 (Suppl 1) S55-\$63. 